\title{
Bioinformatics analysis of differentially expressed miRNA-related mRNAs and their prognostic value in breast carcinoma
}

\author{
GUO-MING ZHANG ${ }^{1,2}$, HEMANT GOYAL $^{3 *}$ and LEI-LEI SONG ${ }^{4 *}$ \\ ${ }^{1}$ Department of Laboratory Medicine, Shuyang People's Hospital; ${ }^{2}$ Department of Laboratory Medicine, \\ Shuyang Affiliated Hospital of Xuzhou Medical University, Shuyang, Jiangsu 223600, P.R. China; \\ ${ }^{3}$ Department of Internal Medicine, Mercer University School of Medicine, Macon, GA 31201, USA; \\ ${ }^{4}$ Department of Laboratory Medicine, The 82th Hospital of the Chinese People's \\ Liberation Army, Huai'an, Jiangsu 223001, P.R. China
}

Received November 14, 2017; Accepted April 17, 2018

DOI: $10.3892 /$ or.2018.6393

\begin{abstract}
Breast carcinoma is one of the most common types of malignant neoplasms, and is associated with high rates of morbidity and mortality. Altered gene expression is critical in the development of breast cancer. To identify the important differentially expressed genes and microRNAs in breast carcinoma, mRNA (GSE26910, GSE42568, and GSE89116) and microRNA (GSE35412) microarray datasets were downloaded from the Gene Expression Omnibus database. The differentially expressed microRNA expression data were extracted with GEO2R online software. The DAVID online database was used to perform a function and pathway enrichment analysis of the key identified differentially expressed genes. A proteinprotein interaction (PPI) network was constructed using the STRING online database, and visualized in Cytoscape software. The effect of the expression level of the key identified genes on overall survival (OS) time was analyzed by using the Kaplan-Meier Plotter online database. Furthermore, the online miRNA databases TargetScan, microT-CDS, and TarBase were used to identify the target genes of the differentially expressed miRNAs. A total of 254 differentially expressed genes were
\end{abstract}

Correspondence to: Dr Guo-Ming Zhang, Department of Laboratory Medicine, Shuyang People's Hospital, 9 Yingbin Road, Shuyang, Jiangsu 223600, P.R. China

E-mail: zly52120@163.com

Dr Lei-Lei Song, Department of Laboratory Medicine, The 82th Hospital of the Chinese People's Liberation Army, 100 Jiankang Dong Road, Huai'an, Jiangsu 223001, P.R. China

E-mail: songleileiyouxiang@163.com

${ }^{*}$ Contributed equally

Key words: breast carcinoma, differentially expressed genes, protein-protein interaction, microRNA, Gene Expression Omnibus, Kaplan-Meier plotter identified, which were enriched in cell adhesion, polysaccharide binding, extracellular region part and ECM-receptor interactions. The PPI network contained 250 nodes and 375 edges. Five differentially expressed genes were found to be significantly negatively correlated with the differentially expressed miRNAs, which were potentially also target genes for miRNAs. Four of the five genes, including $A K A P 12, S O P B$, TCF7L2, COL12A1 and TXNIP were downregulated, and were associated with the OS of patients with breast carcinoma. In addition, a total of 130 differentially expressed miRNAs were identified. In conclusion, these results constitute a novel model for miRNA-mRNA differential expression patterns, and further studies may provide potential targets for diagnosing and understanding the mechanisms of breast carcinoma.

\section{Introduction}

Breast cancer is one of the most common types of malignancy among women, and is associated with high mortality. Globally, more than 1.7 million individuals are diagnosed with breast cancer annually, and approximately 521,000 individuals succumb to the disease (1). In recent decades, the incidence of breast cancer has increased, with almost one-tenth of all newly diagnosed cancers worldwide originating in the breast (2). Changes in reproductive and lifestyle characteristics are contributing to the increased morbidity and mortality rates of breast carcinoma. However, the exact molecular mechanisms underlying breast carcinoma are not fully understood.

At present, three major protein markers: estrogen receptor (ER), progesterone receptor (PR) and human epidermal growth factor (EGF) receptor 2 (HER2), are used for determining the classification, treatment and prognosis of breast carcinoma (3). However, there are no identified protein markers for the early diagnosis and treatment of breast carcinoma (4). Therefore, it is necessary to further investigate the molecular regulatory mechanisms of breast carcinoma, and identify molecular markers that can be used for early diagnosis and monitoring.

In recent years, DNA microarray analysis has been developed as a rapid, high-throughput detection technology to simultane- 
ously monitor the differential expression of numerous genes or miRNAs in oncology research, including in the field of breast cancer (5-8). miRNAs are small 21 -nt RNAs involved in posttranscriptional gene regulation. It has been demonstrated that miRNAs pair with the mRNA 3'-untranslated region (UTR) of target genes to regulate their expression, including in cancer. In breast cancer, multiple miRNAs have been identified to regulate the expression of target genes, including miRNA-155, miRNA675, miRNA-519a and miRNA-31 (9-12), among others.

Although DNA microarray application in oncology research has been widely recognized, the test has high variability. Therefore, in our research, we identified differentially expressed genes and microRNAs by analyzing three breast cancer mRNA microarray datasets, and one microRNA dataset. We then aimed to identify the key genes in breast cancer with survival, mRNA-microRNA interaction, ontology enrichment and network analyses.

\section{Materials and methods}

Microarray data. The GEO (https://www.ncbi.nlm.nih.gov/ gds. Accessed Jan. 26, 2018) is a free international public repository of high-throughput functional genomics data, including microarray and next-generation sequencing data. In this study, we used three gene expression profiles (GSE26910, GSE42568 and GSE89116) and one miRNA expression profile (GSE35412) from GEO.

The GSE26910 dataset was comprised of six breast cancer and six para-carcinoma tissue sample mRNA expression profiles (13); GSE42568 included 104 breast cancer and 17 paracarcinoma tissue sample mRNA expression profiles (14); GSE89116 contained 30 breast cancer and nine para-carcinoma tissue sample mRNA expression profiles (15); and GSE35412 was comprised of 29 breast cancer and 21 para-carcinoma tissue sample miRNA expression profiles (16). All datasets were downloaded in a processed and normalized format.

Data processing. The GEO2R (https:/www.ncbi.nlm.nih.gov/ geo/geo2r. Accessed Jan. 26, 2018) is an online data analysis tool that can be utilized to analyze GEO data series obtained under the same experimental conditions (17). In this study, GEO2R was used to identify the differentially expressed miRNAs and genes between the breast cancer and para-carcinoma tissue expression profiles. Adjusted P-values (adj.p) were calculated using the Benjamini and Hochberg false discovery rate method to correct for the likelihood of false positive results. An adj.P $<0.01$ and $|\operatorname{logFC}|>1$ were set as the cut-off criteria for differential expression.

Functional and pathway enrichment analysis of the differentially expressed genes. The DAVID (https://david-d.ncifcrf. gov/summary.jsp. Accessed Jan. 26, 2018) online database is an online program that provides comprehensive gene annotation tools (18). The database was used to perform gene ontology (GO) and Kyoto Encyclopedia of Genes and Genomes (KEGG) pathway enrichment analyses. $\mathrm{P}<0.05$ was applied as the cut-off criterion.

Protein-protein interaction (PPI) network construction and module selection for the differentially expressed genes. The

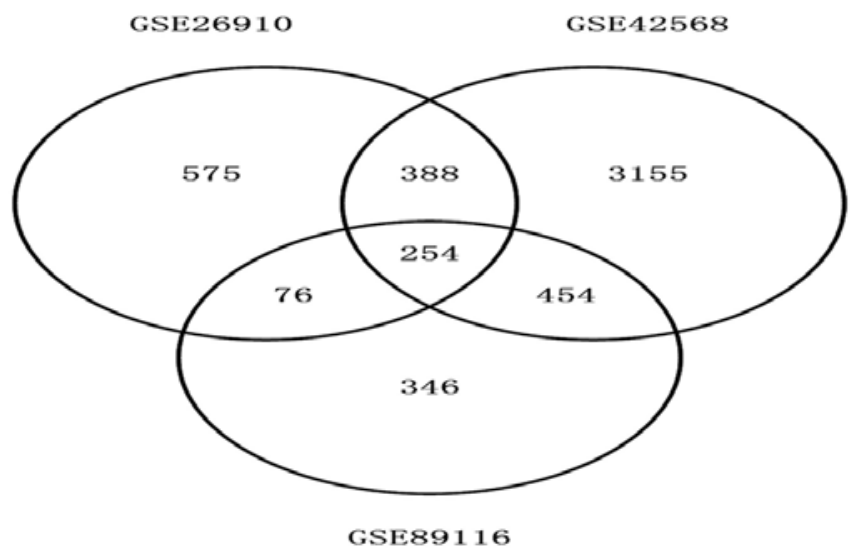

Figure 1. Identification of the differentially expressed genes in the GSE26910, GSE42568 and GSE89116 mRNA microarray datasets.

construction of a network of interactions between proteins can establish a framework for the study of molecular mechanisms. In this study, a PPI network of the differentially expressed genes was constructed using the Search Tool for the Retrieval of Interacting Genes and Proteins (STRING) database (https:// string-db.org. Accessed Jan. 26, 2018) (19), followed by visualization using Cytoscape software (20). The confidence score $\geq 0.7$ was set as the cut-off criterion. The PPI network module selection criteria included a degree cut-off $=2$, node score cut-off $=0.2$, k-core $=2$ and maximum depth=100 (21).

Prediction of miRNA target genes. The online databases TargetScan (http://www.targetscan.org. Accessed Jan. 26, 2018), microT-CDS (http://diana.imis.athena-innovation.gr/DianaTools/index.php?r=microT_CDS. Accessed Jan. 26, 2018) and Tarbase (http://diana.imis.athena-innovation.gr/DianaTools/index.php. Accessed Jan. 26, 2018) were used to identify the target genes of the differentially expressed miRNAs. These databases are universally recognized for their ability to accurately predict miRNA target genes.

Analysis of the effect of the differentially expressed genes on overall survival (OS). In the online database, KaplanMeier (KM) Plotter (http://www.kmplot.com. Accessed Jan. 26, 2018), the impact of 54,675 genes on the survival time of cancer patients was evaluated by analyzing the data from 10,188 cancer samples, including 4,142 breast, 1,648 ovarian, 2,437 lung and 1,065 gastric cancer sample microarray expression profiles (22). In the present study, we divided breast carcinoma patients into two groups depending on the expression of specific genes (high vs. low expression). The KM Plotter database was utilized to analyze the OS of breast cancer patients. Hazard ratios (HR) with $95 \%$ confidence intervals (CI) and a log-rank P-value were calculated and displayed.

\section{Results}

Identification of differentially expressed genes in three GEO datasets. The total number of differentially expressed genes was 1,293, 4,251 and 1,130 from GSE26910, GSE42568 and GSE89116, respectively. A total of 254 genes showed the same expression trend in all three data sets (Fig. 1). This 


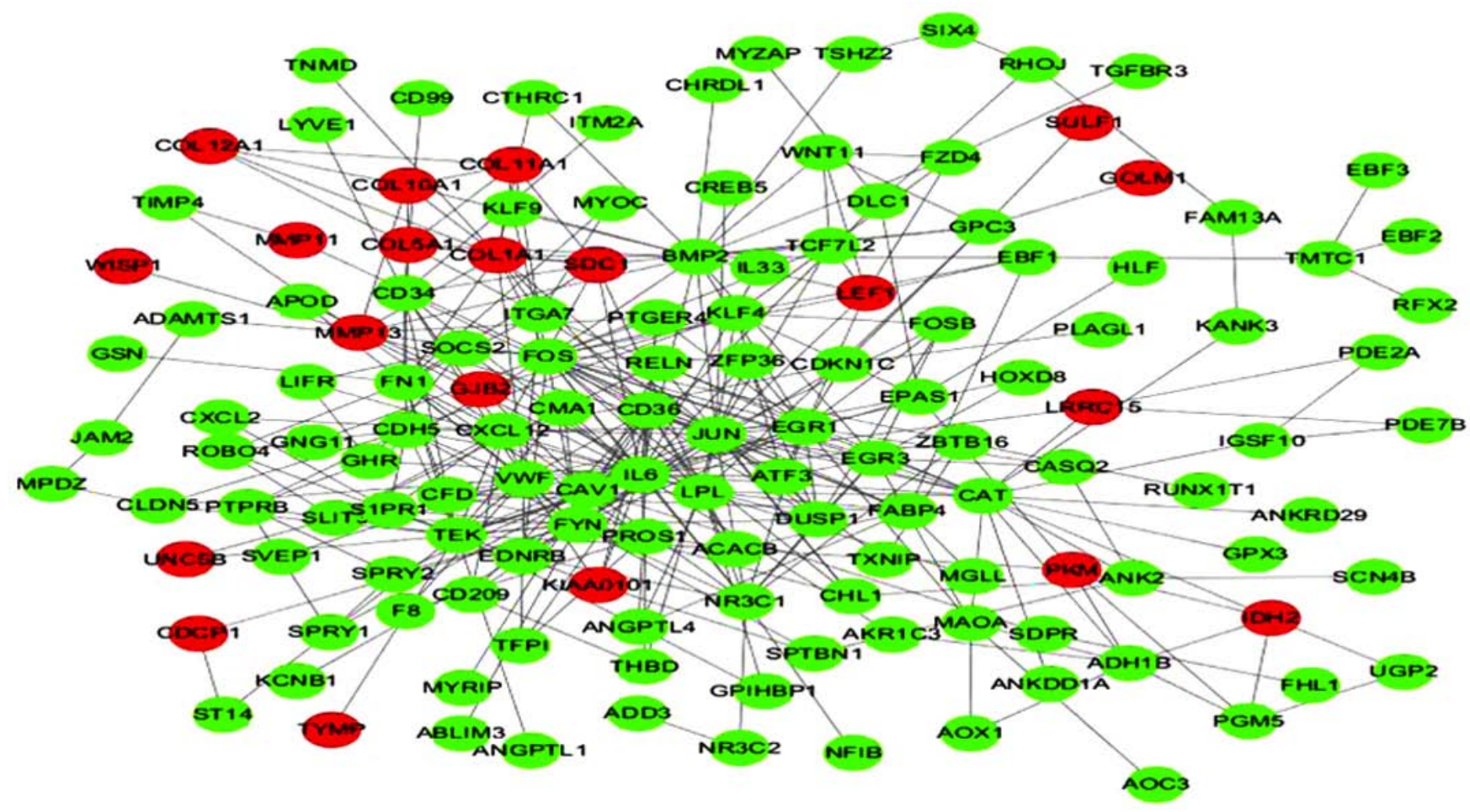

Figure 2. Protein-protein interaction network of the differentially expressed genes. Red nodes represent upregulated genes, while green nodes represent downregulated genes. The edges represent interactions between nodes.

A

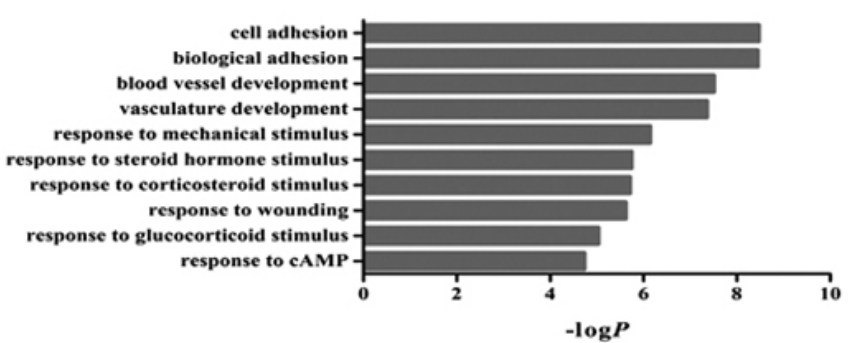

C

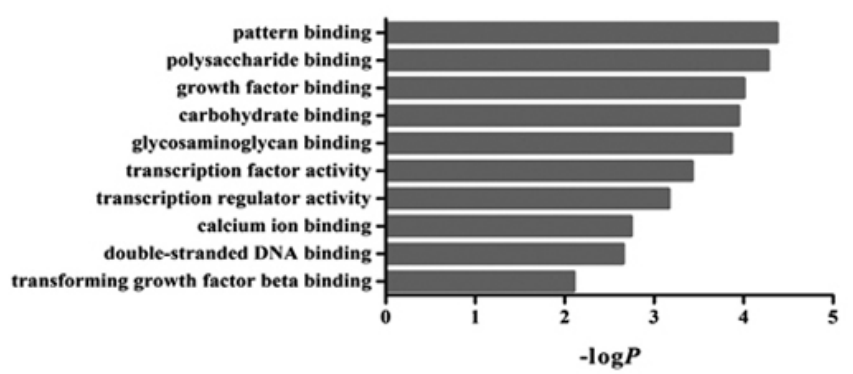

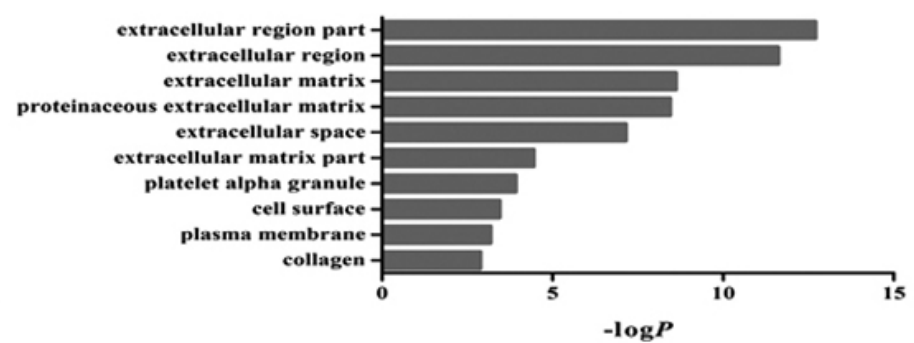

D

KEGG pathway

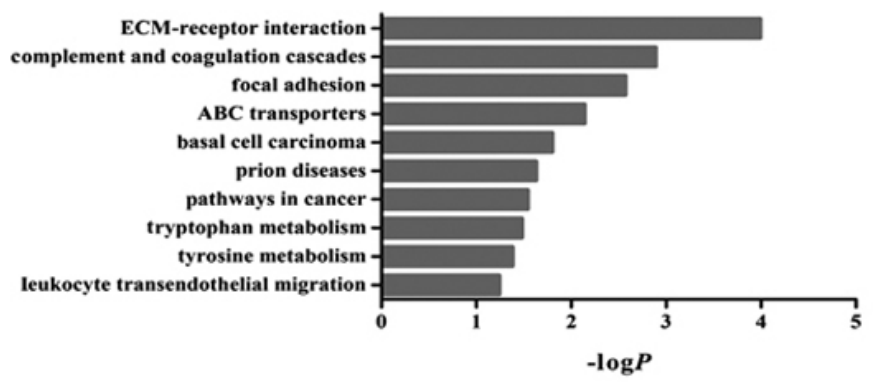

Figure 3. Function and pathway enrichment analysis of upregulated and downregulated genes in breast carcinoma. The gene ontology enrichment analysis covered three categories: (A) biological process, (B) cellular component and (C) molecular function. (D) KEGG analysis suggested that genes were predominately associated with the ECM-receptor interaction pathway. $\mathrm{P}<0.05$ was used as a cut-off for significance.

included 44 upregulated and 210 downregulated differentially expressed genes.

Construction of a PPI network of the differentially expressed genes. The PPI network of the differentially expressed genes consisted of 250 nodes and 375 edges, including 20 upregulated and 139 downregulated genes (Fig. 2).
Function and pathway enrichment analysis. We used the DAVID database to identify enriched functions and pathways of the differentially expressed genes, in order to further understand their function. The differentially expressed genes were the most significantly enriched in cell adhesion (biological process category), polysaccharide binding (molecular function category) and extracellular region part (cellular component 


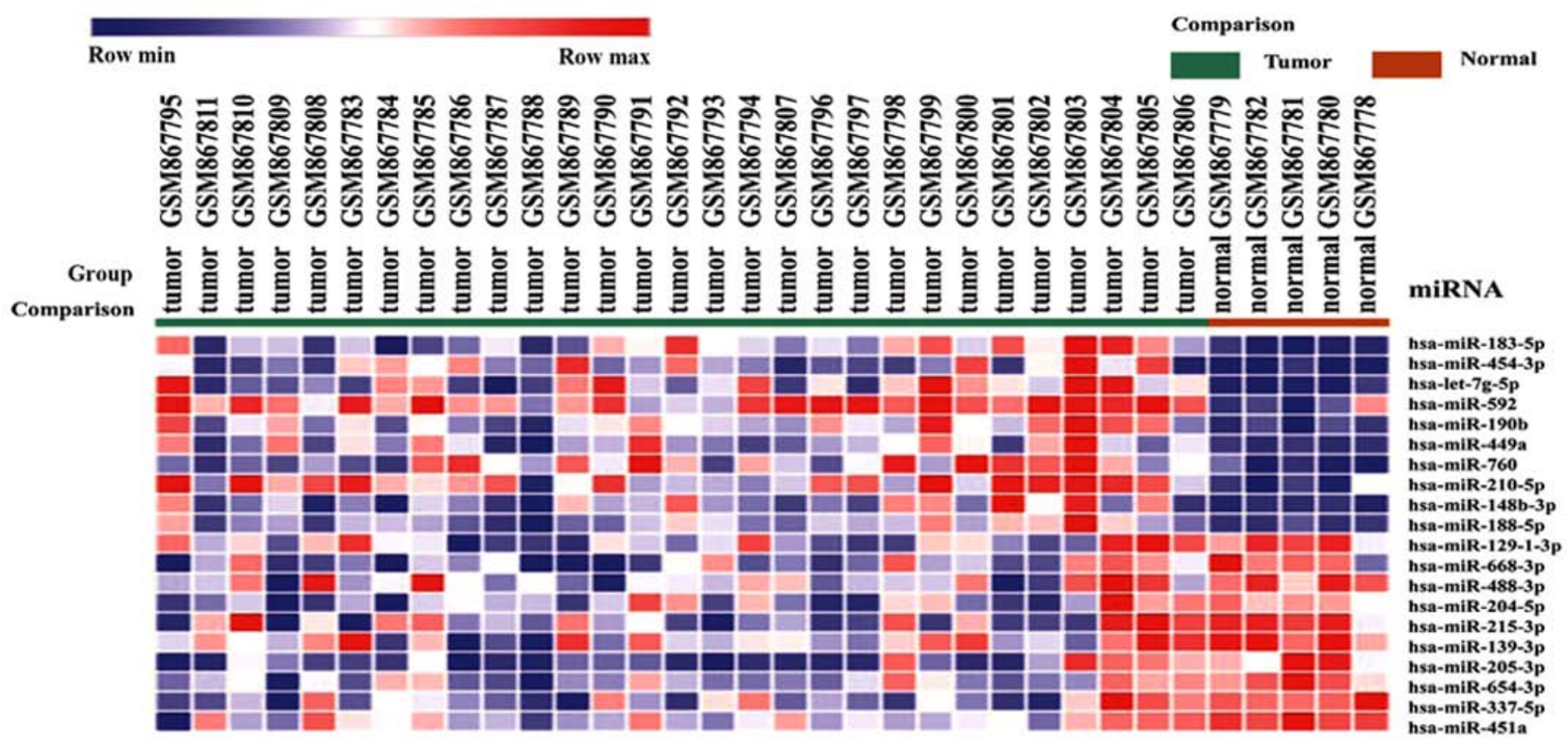

Figure 4. Unsupervised hierarchical clustering analysis using the differentially expressed microRNAs that separated normal and tumor breast tissues. The heat map (based on Spearman's correlation and Euclidean distance) contains log-transformed $\Delta \mathrm{Ct}$ values. Heat map colors correspond to microRNA expression as indicated in the color key: red (upregulated) and blue (downregulated). Orange line, para-carcinoma samples; green line, tumor samples.

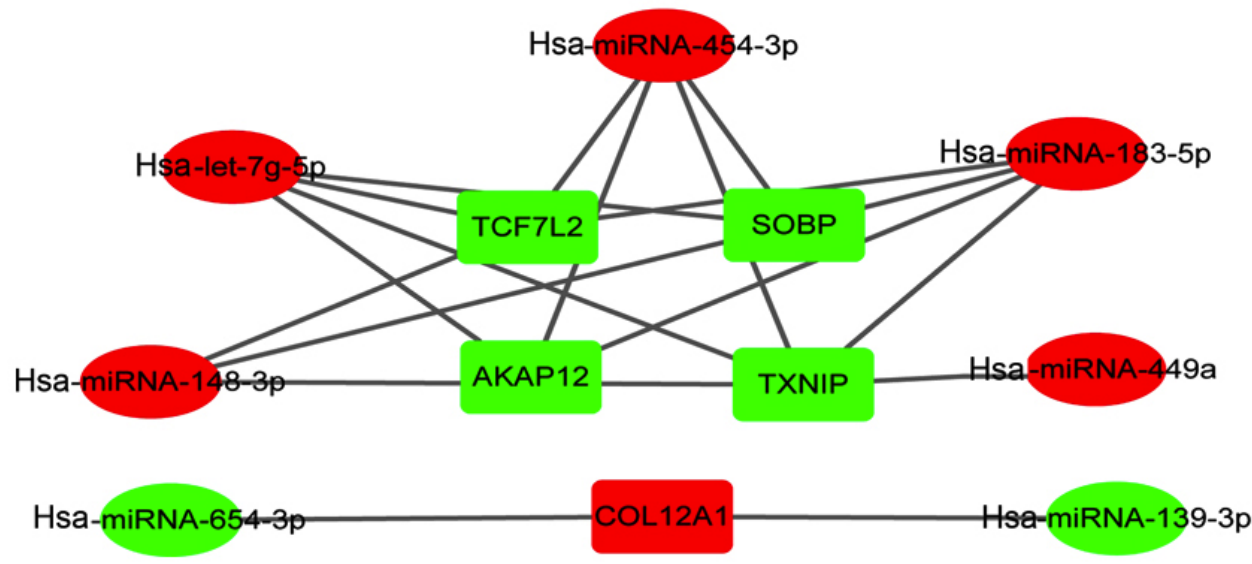

Figure 5. Co-expression networks of overexpressed miRNAs and their target genes in breast carcinoma. Red nodes represent upregulated genes, while green nodes represent downregulated genes.

category) (Fig. 3). In addition, these genes were significantly associated with 10 KEGG pathways, including ECM-receptor interactions, complement and coagulation cascades and adhesion spots (Fig. 3).

Prediction of the target genes of the differentially expressed miRNAs. A total of 130 differentially expressed miRNAs were detected in the GSE35412 dataset, including 17 upregulated and 113 downregulated miRNAs. miR-183-5p was the most significantly upregulated miRNA, whereas miR-129-1-3p was the most significantly downregulated (Fig. 4). Subsequently, target genes of the differentially expressed miRNAs were obtained from online databases (TargetScan, microT-CDS and Tarbase). We determined that AKAP12, SOPB, TCF7L2, COL12A1 and TXNIP were the target genes for multiple differentially expressed miRNAs (Fig. 5).
Survival analysis of key identified differentially expressed genes. The prognostic values of five key genes in the PPI network were assessed from KMplots. The OS rate for breast cancer patients was analyzed based on the low and high expression of the key genes. The results showed that high AKAP12 mRNA expression [HR 0.67 (95\% CI, 0.58-0.79), $\mathrm{P}=6.1 \mathrm{e}-07]$ was associated with an improved OS for breast carcinoma patients (Fig. 6), as was high expression of TXNIP [HR 0.66 (95\% CI, 0.59-0.74), P=1e-13], SOBP [HR 0.83 (95\% CI, 0.74-0.92), $\mathrm{P}=0.00059]$ and TCF7L2 [HR 0.76 (95\% CI, 0.68-0.85), $\mathrm{P}=6 \mathrm{e}-07]$ (Fig. 6).

\section{Discussion}

Although our understanding of the pathogenesis and clinical treatment of breast cancer has made significant progress, 

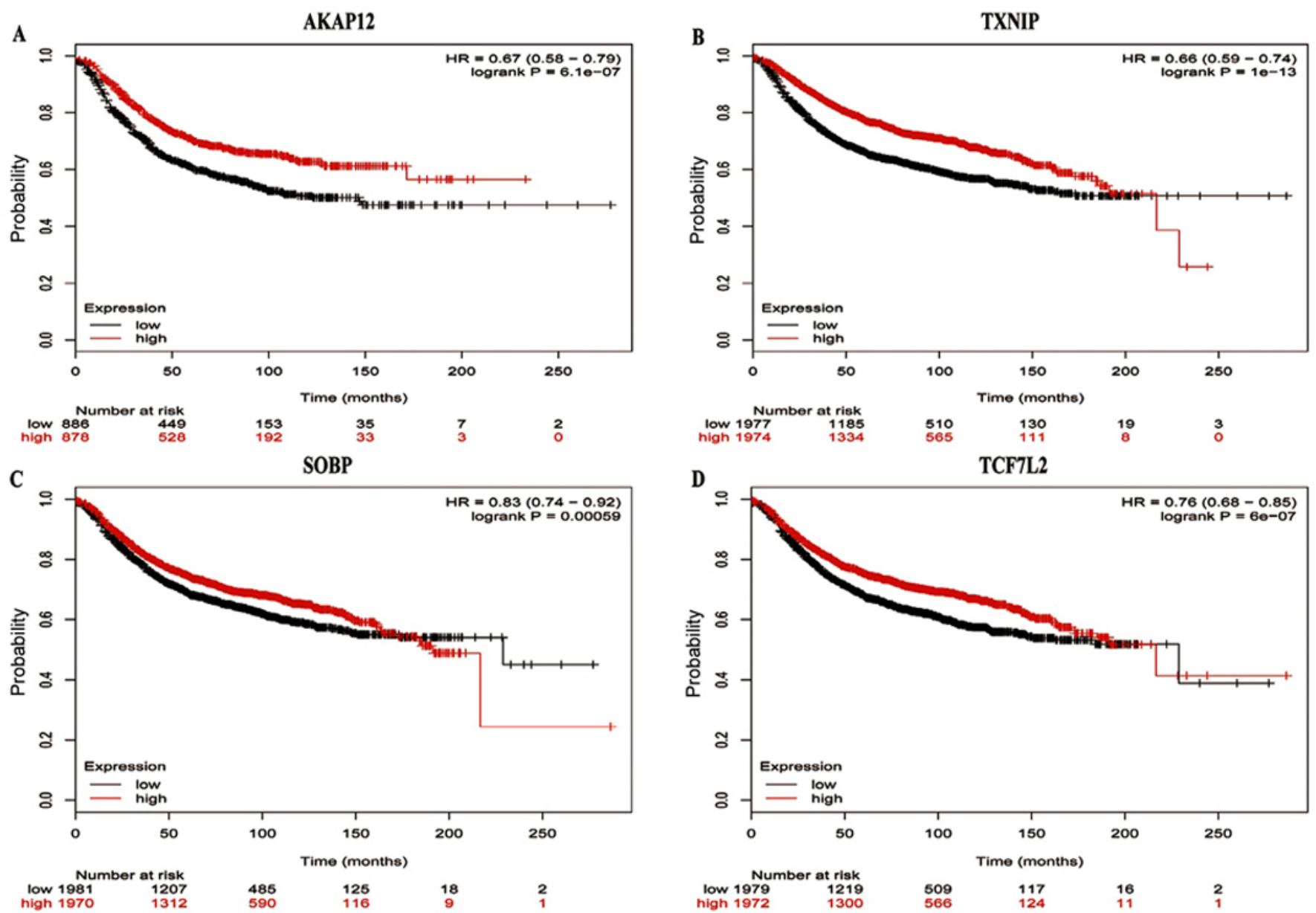

Figure 6. Prognostic value of four genes in breast carcinoma patients. Plots of the prognostic value of (A) AKAP12, (B) TXNIP, (C) SOBP and (D) TCF7L2 genes were obtained from www.kmplot.com. The corresponding Affymetrix IDs were 227529_s_at (AKAP12), 201010_s_at (TXNIP), 218974_at (SOBP) and 216037_x_at (TCF7L2).

the overall mortality rate for breast cancer has not improved significantly, which can be attributed to the lack of molecular markers for effective diagnosis and treatment. Therefore, it is important to explore the molecular markers of breast carcinoma to improve the survival rate and prevention of patients.

Microarray technology has been applied to detect the genetic changes associated with the progression of various types of malignancy. Microarray technology has also been widely used to select molecular markers for determining the diagnosis, treatment and prognosis of tumors. In this study, we screened a total of 254 differentially expressed genes through analyzing three mRNA datasets, which included 44 upregulated genes and 210 downregulated genes. These differentially expressed genes were significantly enriched in cell adhesion, polysaccharide binding, extracellular region part and ECM-receptor interaction, which are all associated with the pathogenesis of carcinomas.

We also analyzed a miRNA dataset, in which we identified 130 differentially expressed miRNAs, including 17 upregulated and 113 downregulated miRNAs, in breast carcinoma. miR183-5p was the most significantly upregulated miRNA, while miR-129-3p was the most markedly downregulated miRNA. miRNAs are small, non-coding RNAs of $\sim 22$ nucleotides in length, which regulate gene expression by targeting the
3'UTR of target mRNAs, resulting in their degradation or the inhibition of translation. Previous research has suggested that the dysregulation of miRNAs is involved in the pathogenesis of many types of cancer, including breast carcinoma. For example, it has been demonstrated that miR-21, miR-210 and miR-221 are upregulated in triple-negative primary breast carcinomas (23). In addition, the overexpression of miR-301 is considered a negative prognostic index for lymph nodenegative invasive ductal breast carcinoma. Shi et al identified that miR-301 is a crucial oncogene in breast carcinoma that promotes nodal and distant relapse via multiple pathways and mechanisms (24).

As miRNAs negatively regulate the expression of their target genes, we analyzed the correlation of upregulated genes with downregulated miRNAs, downregulated genes and upregulated miRNAs. Notably, we identified that $A K A P 12, S O P B$, TCF7L2 and TXNIP were potentially the common targets of hsa-miRNA-183-5p, hsa-miRNA-454-3p and hsa-let-7g-5p among the downregulated genes. COL12A1 was potentially the common target of hsa-miRNA-139-3p and hsa-miRNA654-3p among the upregulated genes. Subsequently, survival analysis of the relationship between the postoperative survival of patients and the expression of these genes suggested that four genes were closely associated with improved OS of breast 
carcinoma patients, namely $A K A P 12, S O P B, T C F 7 L 2$ and TXNIP.

Tumor progression is induced by genetic mutations and epigenetic changes. Protein kinase A anchor 12 (AKAP12) is a scaffold protein that plays a major regulatory role in cell proliferation, migration, apoptosis and angiogenesis (25). Studies have also shown that AKAP12 is involved in multiple signaling pathways through regulating protein kinases $\mathrm{A}$ and C. AKAP12 is a tumor metastasis inhibitory factor, which is associated with carcinoma susceptibility and tumor cell behavior in a variety of tumor types, including breast carcinoma (26-28). AKAP12 has also been associated with miRNAs in various types of disease. In liver cirrhosis, the interaction of miRNA-183 or miRNA-186 can downregulate the expression of AKAP12 (29). In addition, Xia et al indicated that the overexpression of miR-103 can promote cell proliferation and inhibit apoptosis by downregulating AKAP12 expression in hepatocellular carcinoma cell lines (30).

SopB (also known as SigD) is an effector of the Salmonella typhimurium Type III secretion system that acts on phospholipids in the host cell membrane $(31,32)$. SopB may induce epithelial-mesenchymal transition (EMT), which is also associated with malignant disease. SopB plays a central role in activating $\mathrm{Wnt} / \beta$-catenin signaling, which can induce cell transformation and $\mathrm{Wnt} / \beta$-linked regulatory signaling transduction. SopB-dependent activation of Akt kinases can lead to the inhibitory phosphorylation of GSK $3 \beta$, which further induces cytosolic $\beta$-catenin and $\mathrm{Wnt} / \beta$-catenin-mediated EMT (33).

The transcription factor 7-like 2 (TCF7L2) gene is located on the long arm of chromosome 10q25.2 (previously called TCF-4). TCF7L2 is a part of the Wnt/ $\beta$-catenin signaling pathway, which plays an important role in the regulation of cell development and growth $(34,35)$. In addition, epidemiological studies have shown that TCF7L 2 gene polymorphisms are associated with increased susceptibility to carcinomas, including of the breast $(35,36)$. Additionally, TCF7L2 can increase the expression of genes involved in the proliferation, apoptosis, invasion and metastasis of tumor cells $(37,38)$. TCF7L2 synthesis is directly regulated by miR-21 transcription (39). In cervical carcinoma, the expression levels of miR-328 and TCF7L2 are negatively correlated. Furthermore, miR-328 can reduce the expression of TCF7L2 to affect the treatment of cervical carcinoma (40). Cervical carcinoma metastasis and progression may also be inhibited by miR-212, through its direct targeting of TCF7L2 expression (41). In addition, miR181a-5p may regulate the Wnt signaling pathway through the direct targeting of TCF7L2, promoting 3T3-L1 preadipocyte differentiation and adipogenesis (42).

TXNIP (also known as VDUP-1 or TBP-2) is proapoptotic, and inhibits growth and metastasis (43). TXNIP has a variety of functions, including an important regulatory role in the redox equilibrium, and can increase the production of reactive oxygen species (ROS) to induce apoptosis through oxidative stress. TXNIP is a major tumor suppressor gene that is downregulated in a variety of solid tumors, including breast carcinoma (44-46). There is a correlation between the expression of TXNIP and the metastasis and survival prognosis of breast carcinoma (45). TXNIP also plays a critical role in the treatment of HER-1/HER-2-positive tumors, and is a potential prognostic indicator in breast carcinoma (47). TXNIP has been associated with a variety of miRNAs and has been demonstrated as a target of miR-342 (48), miR-135a (49) and miR-20a (50). The miR-373 expression is negatively correlated with the expression of TXNIP, and activation of the miR-373-TXNIP signal transduction axis is associated with a poor outcome in breast carcinoma (51). This may be mediated through an effect on the invasion and migration of breast carcinoma cells, which is associated with the prognosis of breast carcinoma patients (52).

In summary, the present study intended to identify the differentially expressed genes in breast carcinoma and thus find the potential biomarkers for predicting disease progression using comprehensive bioinformatic analyses. In this study, a total of 254 differentially expressed genes and 130 differentially expressed miRNAs were screened; $A K A P 12$, $S O P B, T C F 7 L 2$ and TXNIP, and several miRNAs, including miR-183-5p, let-7g-5p and miR-454-3p, may be key breast carcinoma-associated genes. Our results suggested that data mining and integration is a useful tool to predict the progression of breast carcinoma, and to identify the mechanisms of the occurrence and development of tumors. To apply these gene expression profiles in clinical practice, it is necessary to improve the reliability and reproducibility of this model within dependent datasets in the future. Nevertheless, our research may provide new information for the diagnosis and treatment of breast carcinoma patients.

\section{Acknowledgements}

Not applicable.

\section{Funding}

No funding was received.

\section{Availability of data and materials}

The datasets used during the present study are available from the corresponding author upon reasonable request.

\section{Authors' contributions}

GMZ and LLS conceived and designed the study. HG was involved in the conception of the study. GMZ and LLS wrote the paper. GMZ reviewed and edited the manuscript. GMZ, HG and LLS read and approved the manuscript and agree to be accountable for all aspects of the research in ensuring that the accuracy or integrity of any part of the work is appropriately investigated and resolved.

\section{Ethics approval and consent to participate}

The study was approved by the Institutional Review Board of the Department of Shuyang People's Hospital (Shuyang, China).

\section{Consent for publication}

Not applicable. 


\section{Competing interests}

The authors declare that they have no competing interests.

\section{References}

1. Goldhirsch A, Winer EP, Coates AS, Gelber RD, PiccartGebhart M, Thürlimann B, Senn HJ, Albain KS, André F, Bergh J, et al; Panel members: Personalizing the treatment of women with early breast cancer: Highlights of the St Gallen International Expert Consensus on the Primary Therapy of Early Breast Cancer 2013. Ann Oncol 24: 2206-2223, 2013.

2. Torre LA, Bray F, Siegel RL, Ferlay J, Lortet-Tieulent J and Jemal A: Global cancer statistics, 2012. CA Cancer J Clin 65 . $87-108,2015$

3. Coates AS, Winer EP, Goldhirsch A, Gelber RD, Gnant M, Piccart-Gebhart M, Thürlimann B and Senn HJ; Panel Members: Tailoring therapies - improving the management of early breast cancer: St Gallen International Expert Consensus on the Primary Therapy of Early Breast Cancer 2015. Ann Oncol 26: 1533-1546, 2015.

4. Mohammed ZM, McMillan DC, Edwards J, Mallon E, Doughty JC, Orange C and Going JJ: The relationship between lymphovascular invasion and angiogenesis, hormone receptors, cell proliferation and survival in patients with primary operable invasive ductal breast cancer. BMC Clin Pathol 13: 31, 2013.

5. Mok SC, Bonome T, Vathipadiekal V, Bell A, Johnson ME, Wong KK, Park DC, Hao K, Yip DK, Donninger H, et al: A gene signature predictive for outcome in advanced ovarian cancer identifies a survival factor: Microfibril-associated glycoprotein 2. Cancer Cell 16: 521-532, 2009.

6. Bowen NJ, Walker LD, Matyunina LV, Logani S, Totten KA, Benigno BB and McDonald JF: Gene expression profiling supports the hypothesis that human ovarian surface epithelia are multipotent and capable of serving as ovarian cancer initiating cells. BMC Med Genomics 2: 71, 2009.

7. Vilming Elgaaen B, Olstad OK, Haug KB, Brusletto B Sandvik L, Staff AC, Gautvik KM and Davidson B: Global miRNA expression analysis of serous and clear cell ovarian carcinomas identifies differentially expressed miRNAs including miR-200c-3p as a prognostic marker. BMC Cancer 14: 80, 2014.

8. Elgaaen BV, Olstad OK, Sandvik L, Odegaard E, Sauer T, Staff AC and Gautvik KM: ZNF385B and VEGFA are strongly differentially expressed in serous ovarian carcinomas and correlate with survival. PLoS One 7: e46317, 2012.

9. Kong W, He L, Richards EJ, Challa S, Xu CX, Permuth-Wey J, Lancaster JM, Coppola D, Sellers TA, Djeu JY, et al: Upregulation of miRNA-155 promotes tumour angiogenesis by targeting VHL and is associated with poor prognosis and triple-negative breast cancer. Oncogene 33: 679-689, 2014.

10. Vennin C, Spruyt N, Dahmani F, Julien S, Bertucci F, Finetti P, Chassat T, Bourette RP, Le Bourhis X and Adriaenssens E: H19 non coding RNA-derived miR-675 enhances tumorigenesis and metastasis of breast cancer cells by downregulating c-Cbl and Cbl-b. Oncotarget 6: 29209-29223, 2015.

11. Ward A, Shukla K, Balwierz A, Soons Z, König R, Sahin O and Wiemann S: MicroRNA-519a is a novel oncomir conferring tamoxifen resistance by targeting a network of tumour-suppresso genes in $\mathrm{ER}^{+}$breast cancer. J Pathol 233: 368-379, 2014.

12. Rasheed SA, Teo CR, Beillard EJ, Voorhoeve PM, Zhou W, Ghosh S and Casey PJ: MicroRNA-31 controls G protein alpha-13 (GNA13) expression and cell invasion in breast cancer cells. Mol Cancer 14: 67, 2015.

13. Planche A, Bacac M, Provero P, Fusco C, Delorenzi M, Stehle JC and Stamenkovic I: Identification of prognostic molecular features in the reactive stroma of human breast and prostate cancer. PLoS One 6: e18640, 2011

14. Clarke C, Madden SF, Doolan P, Aherne ST, Joyce H, O'Driscoll L, Gallagher WM, Hennessy BT, Moriarty M, Crown J, et al: Correlating transcriptional networks to breast cancer survival: A large-scale coexpression analysis. Carcinogenesis 34: 2300$2308,2013$.

15. Wasson MK, Chauhan PS, Singh LC, Katara D, Dev Sharma J, Zomawia E, Kataki A, Kapur S and Saxena S: Association of DNA repair and cell cycle gene variations with breast cancer risk in Northeast Indian population: A multiple interaction analysis. Tumour Biol 35: 5885-5894, 2014
16. Romero-Cordoba S, Rodriguez-Cuevas S, Rebollar-Vega R, Quintanar-Jurado V, Maffuz-Aziz A, Jimenez-Sanchez G, Bautista-Piña V, Arellano-Llamas R and Hidalgo-Miranda A: Identification and pathway analysis of microRNAs with no previous involvement in breast cancer. PLoS One 7: e31904, 2012

17. Barrett T, Wilhite SE, Ledoux P, Evangelista C, Kim IF Tomashevsky M, Marshall KA, Phillippy KH, Sherman PM, Holko M, et al: NCBI GEO: Archive for functional genomics data sets - update. Nucleic Acids Res 41: D991-D995, 2013.

18. Huang W, Sherman BT and Lempicki RA: Systematic and integrative analysis of large gene lists using DAVID bioinformatics resources. Nat Protoc 4: 44-57, 2009.

19. Szklarczyk D, Franceschini A, Wyder S, Forslund K, Heller D, Huerta-Cepas J, Simonovic M, Roth A, Santos A, Tsafou KP, et al: STRING v10: Protein-protein interaction networks, integrated over the tree of life. Nucleic Acids Res 43: D447-D452, 2015.

20. Shannon P, Markiel A, Ozier O, Baliga NS, Wang JT, Ramage D, Amin N, Schwikowski B and Ideker T: Cytoscape: A software environment for integrated models of biomolecular interaction networks. Genome Res 13: 2498-2504, 2003.

21. Bader GD and Hogue CW: An automated method for finding molecular complexes in large protein interaction networks. BMC Bioinformatics 4: 2, 2003.

22. Gyorffy B, Lánczky A and Szállási Z: Implementing an online tool for genome-wide validation of survival-associated biomarkers in ovarian-cancer using microarray data from 1287 patients. Endocr Relat Cancer 19: 197-208, 2012.

23. Radojicic J,Zaravinos A, Vrekoussis T, Kafousi M, Spandidos DA and Stathopoulos EN: MicroRNA expression analysis in triplenegative (ER, PR and Her2/neu) breast cancer. Cell Cycle 10 507-517, 2011.

24. Shi W, Gerster K, Alajez NM, Tsang J, Waldron L, Pintilie M, Hui AB, Sykes J, P'ng C, Miller N, et al: MicroRNA-301 mediates proliferation and invasion in human breast cancer. Cancer Res 71: 2926-2937, 2011.

25. Hayashi M, Nomoto S, Kanda M, Okamura Y, Nishikawa Y, Yamada S, Fujii T, Sugimoto H, Takeda S and Kodera Y: Identification of the A kinase anchor protein 12 (AKAP12) gene as a candidate tumor suppressor of hepatocellular carcinoma. J Surg Oncol 105: 381-386, 2012

26. Flotho C, Paulun A, Batz C and Niemeyer CM: AKAP12, a gene with tumour suppressor properties, is a target of promoter DNA methylation in childhood myeloid malignancies. Br J Haematol 138: 644-650, 2007.

27. Su B, Zheng Q, Vaughan MM, Bu Y and Gelman IH: SSeCKS metastasis-suppressing activity in MatLyLu prostate cancer cells correlates with vascular endothelial growth factor inhibition. Cancer Res 66: 5599-5607, 2006.

28. Choi MC, Jong HS, Kim TY, Song SH, Lee DS, Lee JW, Kim TY, Kim NK and Bang YJ: AKAP12/Gravin is inactivated by epigenetic mechanism in human gastric carcinoma and shows growth suppressor activity. Oncogene 23: 7095-7103, 2004.

29. Goeppert B, Schmezer P, Dutruel C, Oakes C, Renner M, Breinig M, Warth A, Vogel MN, Mittelbronn M, Mehrabi A, et al: Down-regulation of tumor suppressor A kinase anchor protein 12 in human hepatocarcinogenesis by epigenetic mechanisms. Hepatology 52: 2023-2033, 2010.

30. Xia W, Ni J, Zhuang J, Qian L, Wang P and Wang J: MiR-103 regulates hepatocellular carcinoma growth by targeting AKAP12. Int J Biochem Cell Biol 71: 1-11, 2016.

31. Ruan HH, Li Y, Zhang XX, Liu Q, Ren H, Zhang KS and Zhao H: Identification of TRAF6 as a ubiquitin ligase engaged in the ubiquitination of SopB, a virulence effector protein secreted by Salmonella typhimurium. Biochem Biophys Res Commun 447: 172-177, 2014.

32. Perrett CA and Zhou D: Erratum: Salmonella type III effector SopB modulates host cell exocytosis. Emerg Microbes Infect 2: e39, 2013

33. Tahoun A, Mahajan S, Paxton E, Malterer G, Donaldson DS, Wang D, Tan A, Gillespie TL, O'Shea M, Roe AJ, et al: Salmonella transforms follicle-associated epithelial cells into $\mathrm{M}$ cells to promote intestinal invasion. Cell Host Microbe 12: 645-656, 2012

34. Ravindranath A, O'Connell A, Johnston PG and El-Tanani MK: The role of LEF/TCF factors in neoplastic transformation. Curr Mol Med 8: 38-50, 2008.

35. Connor AE, Baumgartner RN, Baumgartner KB, Kerber RA, Pinkston C, John EM, Torres-Mejia G, Hines L, Giuliano A, Wolff RK, et al: Associations between TCF7L2 polymorphisms and risk of breast cancer among Hispanic and non-Hispanic white women: The Breast Cancer Health Disparities Study. Breast Cancer Res Treat 136: 593-602, 2012. 
36. Lu XP, Hu GN, Du JQ and Li HQ: TCF7L2 gene polymorphisms and susceptibility to breast cancer: A meta-analysis. Genet Mol Res 14: 2860-2867, 2015.

37. Naidu R, Yip $\mathrm{CH}$ and Taib NA: Genetic variations in transcription factor 7-like 2 (TCF7L2) gene: Association of TCF7L2 rs12255372(G/T) or rs7903146(C/T) with breast cancer risk and clinico-pathological parameters. Med Oncol 29: 411-417, 2012.

38. Min W, Liu X, Lu Y, Gong Z, Wang M, Lin S, Kang H, Jin T, Wang X, Ma X, et al: Association of transcription factor 7-like 2 gene polymorphisms with breast cancer risk in northwest Chinese women. Oncotarget 7: 77175-77182, 2016.

39. Lan F, Yue X, Han L, Shi Z, Yang Y, Pu P, Yao Z and Kang C: Genome-wide identification of TCF7L2/TCF4 target miRNAs reveals a role for miR-21 in Wnt-driven epithelial cancer. Int J Oncol 40: 519-526, 2012.

40. Wang X and Xia Y: microRNA-328 inhibits cervical cancer cell proliferation and tumorigenesis by targeting TCF7L2. Biochem Biophys Res Commun 475: 169-175, 2016.

41. Zhou C, Tan DM, Chen L, Xu XY, Sun CC, Zong LJ, Han S and Zhang YZ: Effect of miR-212 targeting TCF7L2 on the proliferation and metastasis of cervical cancer. Eur Rev Med Pharmacol Sci 21: 219-226, 2017.

42. Ouyang D, Xu L, Zhang L, Guo D, Tan X, Yu X, Qi J, Ye Y, Liu Q, Ma Y, et al: MiR-181a-5p regulates 3T3-L1 cell adipogenesis by targeting Smad7 and Tcf712. Acta Biochim Biophys Sin (Shanghai) 48: 1034-1041, 2016.

43. Kim SY, Suh HW, Chung JW, Yoon SR and Choi I: Diverse functions of VDUP1 in cell proliferation, differentiation, and diseases. Cell Mol Immunol 4: 345-351, 2007.

44. Zhou J, Yu Q and Chng WJ: TXNIP (VDUP-1, TBP-2): A major redox regulator commonly suppressed in cancer by epigenetic mechanisms. Int J Biochem Cell Biol 43: 1668-1673, 2011.
45. Cadenas C, Franckenstein D, Schmidt M, Gehrmann M, Hermes M, Geppert B, Schormann W, Maccoux LJ, Schug M, Schumann A, et al: Role of thioredoxin reductase 1 and thioredoxin interacting protein in prognosis of breast cancer. Breast Cancer Res 12: R44, 2010.

46. Zhou J and Chng WJ: Roles of thioredoxin binding protein (TXNIP) in oxidative stress, apoptosis and cancer. Mitochondrion 13: 163-169, 2013.

47. Nie W, Huang W, Zhang W, Xu J, Song W, Wang Y, Zhu A, Luo J, Huang G, Wang Y, et al: TXNIP interaction with the Her-1/2 pathway contributes to overall survival in breast cancer. Oncotarget 6: 3003-3012, 2015.

48. Cittelly DM, Das PM, Spoelstra NS, Edgerton SM, Richer JK Thor AD and Jones FE: Downregulation of miR-342 is associated with tamoxifen resistant breast tumors. Mol Cancer 9: 317, 2010.

49. Zhu HJ, Wang DG, Yan J and Xu J: Up-regulation of microRNA135a protects against myocardial ischemia/reperfusion injury by decreasing TXNIP expression in diabetic mice. Am J Transl Res 7: 2661-2671, 2015

50. Li XF, Shen WW, Sun YY, Li WX, Sun ZH, Liu YH, Zhang L, Huang C, Meng XM and Li J: MicroRNA-20a negatively regulates expression of NLRP3-inflammasome by targeting TXNIP in adjuvant-induced arthritis fibroblast-like synoviocytes. Joint Bone Spine 83: 695-700, 2016.

51. Chen D, Dang BL, Huang JZ, Chen M, Wu D, Xu ML, Li R and Yan GR: MiR-373 drives the epithelial-to-mesenchymal transition and metastasis via the miR-373-TXNIP-HIF1 $\alpha$-TWIST signaling axis in breast cancer. Oncotarget 6: 32701-32712, 2015.

52. Wang JG, Zhang LK, Chen YB, Zhang T, Yuan PF and Liu DC: Influence of miR-373 on the invasion and migration of breast cancer and the expression level of target genes TXNIP. J Biol Regul Homeost Agents 29: 367-372, 2015. 